\title{
Efecto de dos enraizadores en tres especies forestales promisorias para la recuperación de suelos
}

\author{
Luz Adriana Giraldo C. ${ }^{1}$, Héctor Fabio Ríos $0 .{ }^{12} \&$ Manuel Francisco Polanco ${ }^{13}$ \\ hectorfabio9@yahoo.com², manuel.polanco@unad.edu.co ${ }^{3}$ \\ ${ }^{1}$ Universidad Nacional Abierta y A Distancia. Escuela de Ciencias Agrícolas, \\ Pecuarias y del Medio Ambiente. CEAD Eje Cafetero, Pereira.
}

\begin{abstract}
Resumen.- La erosión es uno de los principales problemas que afecta los suelos de ladera en el mundo. Algunas de las especies utilizadas en obras de bioingeniería se propagan por medio de estacas, sin embargo no todas enraízan fácilmente, por lo que es necesario utilizar sustancias inductoras de enraizamiento. En este trabajo, evaluamos dos sustancias promotoras de enraizamiento en estacas de mataratón (Gliricidia sepium), nacedero (Trichanthera gigantea) y sauce (Salix humboldtiana). La investigación se realizó en el municipio de Dosquebradas, Risaralda, a una altitud de $1450 \mathrm{~m}$. Los tratamientos consistieron en la aplicación de un enraizador de síntesis (Hormonagro ${ }^{\circledR}$ ), un enraizador natural (extracto de Aloe vera) y un testigo sin aplicación de inductores. Los resultados obtenidos indican que para las tres especies es necesario emplear estimuladores de enraizamiento. El extracto de $A$. Vera produjo un mejor efecto sobre el enraizamiento de las tres especies, siendo más notorio sobre $S$. humboldtiana, 60 días después de la aplicación. La especie T. gigantea no mostró diferencias significativas con respecto a la aplicación de estimulantes de enraizamiento. En cuanto a la tolerancia de las especies al encharcamiento del sustrato de siembra, S. humboldtiana presentó mayor tolerancia mientras que T. gigantea presento los mayores problemas de pudrición.
\end{abstract}

Palabras clave: conservación de suelos, erosión, estaca, enraizamiento, propagación vegetativa

\begin{abstract}
One of the alternatives to prevent and control erosion of soils is bioengineering, where trees are used to increase the resistance of the soil. Most of those trees are propagated from stakes. Nevertheless, since formatting new roots from vegetative parts is not easy, it is necessary to use rooting inducing substances. We evaluate rooting two rooting of three tree species, "matarratón" (Gliricidia sepium), "nacedero" (Trichanthera gigantea) and "willow" (Salix humboldtiana). The research work was carried out in of Dosquebradas, Risaralda at $1450 \mathrm{~m}$. We applied a chemical rooting inducer $\left(\right.$ Hormonagro $^{\circledast}$ ) and a natural rooting inducer (extract of Aloe vera) and a control treatment with no inducers. Our results indicate that rooting was improved by the addition of a stimulator. $A$. vera improved the rooting of the three species, being far better on S. humboldtiana, 60 days after the application. T. gigantea did not show significant differences related with application of rooting inducers. The tolerance of the species to the excess of humidity in the substrate, S. humboldtiana was more tolerant whereas T. gigantea had the major rotting problems due to the excess of water.
\end{abstract}

Key words: erosive process, soil conservation, stakes, rooting, vegetative propagation

\section{Introducción}

La erosión y los movimientos en masa son fenómenos que día a día dejan devastadas grandes áreas y que, en la mayoría de los casos, provocan pérdidas económicas y de vidas humanas. La erosión, definida como la pérdida de la capa superficial del suelo, es ocasionada por malas prácticas de manejo, como la implantación de cultivos en zonas con alto grado de pendiente y prácticas culturales como la deshierba con azadón, el uso intensivo de herbicidas y la siembra en sentido de la pendiente, entre otras. Por su parte, 
los movimientos en masa son provocados generalmente por la deforestación en terrenos inestables. Actualmente se emplean algunas especies vegetales en obras de bioingeniería, con el fin de dar estabilidad a las laderas y prevenir la erosión y sus consecuencias.

La siembra de ciertas especies forestales puede favorecer la recuperación o estabilización de suelos y laderas que han sufrido los efectos de la erosión. Dichas especies deben tener una gran capacidad de regeneración vegetativa, para que además de reproducirse de forma sexual, puedan ser propagadas y regeneradas mediante fragmentación, división celular, o a partir de órganos o partes vegetativas como yemas, bulbillos, tubérculos, estolones y rizomas; es decir, involucrando solamente a un progenitor, sin fusión de gametos ni intercambio genético. La propagación asexual o vegetativa se utiliza para producir una planta que posea el mismo genotipo que la planta madre (planta donadora) y esto es posible porque todas las células de una planta poseen la información necesaria para reproducir la planta entera (Hartmann et al. 1992 en Taiariol 1997), como ocurre con la begonia (Begonia rex Putz), cuyas hojas partidas en trozos y dispuestas sobre la tierra húmeda son capaces de desarrollar raíces y dar origen a nuevos individuos. Algunas especies como el nacedero (Trichanthera gigantea Nees) y el matarratón (Gliricidia sepium (Jacq.) Kunth ex Walp.), pueden generar también nuevas plantas a partir de trozos de tallo (Conif 1986).

A pesar de que en muchas especies vegetales se presentan naturalmente mecanismos de reproducción vegetativa, es posible que mediante intervención humana se hagan más eficientes y se generen nuevos tipos de multiplicación. El éxito de la propagación vegetativa depende de muchos factores como, por ejemplo, el tipo de especie que se quiere reproducir, el método de reproducción vegetativa que se emplee, las características fisiológicas del material a multiplicar, el genotipo empleado y la metodología de manejo utilizada durante el proceso de propagación. (Rodríguez \& Nieto 2002) y el nivel trófico de las células vegetativas (Brousse \& Loussesrt 1980).

La reproducción por medio de estacas permite una rápida obtención de material para siembra de un genotipo uniforme y es muy útil para la reproducción de aquellas especies que son difíciles de propagar por semilla. En la práctica del estacado pueden utilizarse sustancias para estimular el proceso de enraizamiento, favoreciendo la formación de raíces adventicias. En 1935, el descubrimiento del efecto estimulante de las hormonas sobre el enraizamiento de estacas, hizo posible la elaboración de nuevas técnicas de propagación. Se ha encontrado que algunas auxinas, como el ácido indol-butírico (AIB) y el ácido indol acético (AIA), que estimulan la producción de raíces (Villegas 1984, Kramm, 1987).Existen inductores químicos artificiales, elaborados con ácido alfa-naftalenacético (ANA)como ingrediente activo, que son reguladores fisiológicos que actúan sobre los puntos de crecimiento activo de las raíces de las plantas y afectan las divisiones celulares promoviendo la emisión radical. Actualmente se ha despertado gran interés por el uso de estimuladores de enraizamiento de origen natural, como el cristal de sábila (Aloe vera (L.) Burm.f.) y la cocción de las hojas del sauce (Salix humboldtiana Willd.), entre otros (Hartmann \& Kester 1998).

La sábila (A. vera) pertenece a la familia Liliaceae y es originaria de África, desde donde fue introducida al continente americano por Cristóbal Colón, ya que fue traída como medicina para la tripulación. Durante siglos fue utilizada por sus propiedades medicinales $y$ terapéuticas sin ningún entendimiento claro o análisis científico de cada una de sus propiedades (Sánchez 2007). La Sábila es una planta perenne, de rizoma largo, parecido a un maguey, con una raíz alargada que forma un rizoma que puede ser dividido para propagar la planta (Bonn 1836 en Durán 2007). La sábila tiene una amplia adaptación a diferentes climas y suelos, incluso en áreas con baja fertilización y suelos poco fértiles, (Durán 2007).

El gel de A. vera contiene alrededor de $98.5 \%$ de agua, es rico en mucílagos, formados por ácidos galacturónicos, glucorónicos, unidos a azúcares como glucosa, galactosa y arabinosa; también están presentes otrospolisacáridoscon alto contenido en ácidos urónicos, fructuosa y otros azúcares hidrolizables. Químicamente se caracteriza por la presencia de compuestos fenólicos de gran poder antioxidante, que son generalmente clasificados en dos grupos principales; , las cromonas y las antroquinonas 
(Sánchez 2007). Contiene algunas vitaminas hidrosolubles y minerales como calcio, fósforo potasio, hierro, sodio, magnesio, manganeso, cobre, cromo y zinc, además de alrededor de 17 aminoácidos, siendo el principal la Arginina. También presenta enzimas como la oxidasa, catalasa y amilasa (Sánchez, 2007).

En este trabajo buscamos determinar el efecto de la aplicación del extracto de $A$. vera como estimulador de enraizamiento de origen natural, en tres especies forestales promisorias, utilizadas en la prevención y control de procesos erosivos avanzados del suelo.

\section{Materiales y métodos}

El ensayo se llevó a cabo en el Municipio de Dosquebradas, en el departamento de Risaralda, Colombia, en los predios de la Universidad Nacional Abiertra y a Distancia (UNAD) , a una altitud de $1450 \mathrm{~m}$, con temperatura media de $24^{\circ} \mathrm{C}$, precipitación media anual de $2200 \mathrm{~mm}$ y una humedad relativa del $80 \%$. Los suelos de la zona son de origen volcánico.

Utilizamos estacas de tres especies de árboles a las cuales aplicamos dos tipos de enraizadores. Aplicamos ácidonaftalenácetico (Hormonagro ${ }^{\circledR}$ ANA 0.4\%) como enraizador de síntesis, en una solución al 6\% y utilizamos un extracto de cristales de sábila (A. vera) macerados como enraizador natural; en ambos casos el tratamiento fue aplicado el extremo basal de las estacas antes de la siembra. Como control, sembramos estacas de las tres especies sin ningún tipo de entraizador. Adicionalmente, para evitar el ataque de hongos, aplicamos carbendazim $\left(\right.$ Derosal $^{\circledR}$ ) en una dosis del $1 \%$ sobre todos los tratamientos, utilizando un aspersor manual.

Obtuvimos estacas o esquejes de árboles vigorosos mayores de diez años, sanos y con abundancia de rebrotes, cuidando que para cada especie el número de yemas, longitud y diámetro fueran iguales. Sembramos las estacas en bolsas de almácigo con sustrato tierra-cascarilla de arroz en proporción 5:1 y las mantuvios bajo polisombra del $45 \%$. Para la desinfección del sustrato utilizamos formol al $2.5 \%$.

Como variables de respuesta medimos el peso seco de las raíces a los 45 y 60 días después de la siembra y el porcentaje de mortalidad. Empleamos un diseño en bloques completos al azar, con nueve tratamientos y cinco bloques (repeticiones) para un total de 45 unidades experimentales, cada una con seis estacas de la misma especie.

Descripcion de las especies empleadas.- El matarratón (Gliricidia sepium (Jacq.) Kunth ex Walp.) es una especie de árbol de origen Centro y Suramericano, perteneciente a la familia Papilonaceae, que crece ente los diez y los $1800 \mathrm{msnm}$. Hace miles de años era utilizado como un árbol multipropósito por los mayas y desde entonces ha sido utilizado para cercas vivas, sombrío de cultivos, construcciones, forraje para animales, leña, abono verde, estabilización de suelos y como especie ornamental. Después de la leucaena (Leucaena leucocephala), es tal vez el árbol leguminoso más cultivado y en muchos casos puede producir más biomasa que ésta (Stewart et al, 1992 en Parent 1989).

Es un árbol de tamaño pequeño a mediano, que alcanza una altura de 10 a 12 metros, el diámetro basal alcanza entre 50 y $70 \mathrm{~cm}$. Las hojas son pinnadas, usualmente alternas, subopuestas u opuestas, de aproximadamente $30 \mathrm{~cm}$ de longitud, la inflorescencia aparece como racimos agrupados en partes distales de ramas viejas y nuevas de 5 a $15 \mathrm{~cm}$ de largo, conteniendo de 20 a 40 flores por racimo. Las flores son de color morado (Ángel 1990). El matarratón, es capaz de retoñar vigorosamente después de la poda y tolera repetidos cortes; sin embargo, su fenología cambia con los cortes; es así como retiene las hojas en la época seca, cuando generalmente los rebrotes viejos son deciduos. Esto permite que dicha especie provea de forraje en la época seca en los trópicos. (Bos \& Van 1986).

El Sauce (Salix humboldtiana Willd.) es originario de Ámerica subtropical y ahora es cultivado en las zonas tropicales de Suramérica, el sur de los Estados Unidos y las Antillas (Vázquez-Yanes et al. 1999), pertenece a la familia Salicaceae y se adapta muybien a alturas comprendidas entre los 1.400 a $2.400 \mathrm{msnm}$. Es un árbol es mediano que normalmente puede alcanzar entre 15 y 16 metros de altura y 50 a $60 \mathrm{~cm}$ de diámetro en el tronco. Es de copa columnar estrecha y corteza fisurada. Las hojas son simples alternas, espiralazas, lineales, con dos estipulas en la base de las 
hojas de borde finamente aserrado. Las flores unisexuales en árboles diferentes, es dioico. El fruto es una cápsula ovoide de 3 a $6 \mathrm{~cm}$. de largo; la semilla se encuentran rodeadas de pelos blancos, parecidas al algodón. El sauce es importante como estabilizador y protector en las márgenes de ríos y quebradas, o como colonizador en programas de recuperación de áreas deforestadas. Se utiliza para repoblación forestal; se emplea para fines técnicos de establecimiento de barreras vivas, barreras rompevientos, y protección de nacimientos de agua, como sombrío y ornamental.

El nacedero, quiebrabarrigo (Trichanthera gigantea Nees): pertenece a la familia Acanthaceae, es originario del Norte de Suramérica, donde crece en alturas entre el nivel del mar y los $2300 \mathrm{~m}$. Es un árbol de, 5 $\mathrm{m}$ de altura promedio, aunque puede llegar a crecer hasta los $17 \mathrm{~m}$.; tiene un diámetro al pecho de $0,35 \mathrm{~m}$. Es un árbol muy ramificado con follaje verde oscuro, con flores rojas y ramas con nudos muy pronunciados. Esta especie requiere de abundante luz y su semilla presenta inconvenientes para germinar, por lo que su propagación se facilita por medio del estacado. Crece sobre suelos profundos, aireados y de buen drenaje, generalmente cerca de aguas en movimiento. Se ha registrado como alimento de animales además de emplearse en medicina tradicional (Ospina \& Murgueitio 2002).

\section{Resultados}

No encontramos diferencias significativas entre tratamientos en el peso seco de las raíces de G. sepium y S. humboldtiana 45 días después de la siembra, mientras que en $T$. gigantea se presentaron diferencias significativas entre el tratamiento Hormonagro ${ }^{\otimes}$ y el control. No se encontraron diferencias entre el tratamiento con sábila comparado con el control, como tampoco entre el tratamiento con sábila y el producto comercial. (Tabla 1). Sin embargo hubo diferencias en el peso seco de las raíces en los tratamientos con los enraizadotes, que fue mayor que el registrado en el tratamiento de control.

Las diferencias en peso seco de raíces de $T$. gigantea entre tratamientos fueron menores $y$ resultaron altamente significativas, debido posiblemente a que los coeficientes de variación en esta especie fueron bajos. El peso seco promedio de raíces obtenido en el tratamiento con en producto comercial Hormonagro $^{\circledR}$ fue más alto que el obtenido en el testigo; en el tratamiento con sábila el peso de raíces fue mayor comparado con el testigo. El peso seco de raíces registrados en la especie $S$. humboldtiana, fue similar en los tratamientos y el control. En el segundo muestreo, 60 días después de la siembra, no hubo diferencia significativa por efecto de los tratamientos, pero sí por efecto de bloques o réplicas. Esta variación pudo deberse a la pérdida de estacas por factores no controlados como el exceso de humedad (Tabla 1).

Siguiendo el patrón observado durante el primer muestreo para G. sepium, no se presentaron diferencias significativas entre los tratamientos con enraizadores, el peso seco de las raíces en los tratamientos con Hormonagro $^{\circledast}$ y con Sábila fueron mayores que el registrado en el control. En T. gigantea, se encontraron diferencias significativas entre tratamientos y el peso seco promedio de raíces obtenido en el tratamiento con Hormonagro fue más alto que el obtenido en el control; en el tratamiento con Sábila el peso de raíces fue mayor comparado con el control. El peso seco de raíces registrado para $S$. humboldtiana, fue similar en los dos tratamiento y en el testigo.

En la especie G. sepium se presentó una reducción en peso del $27.7 \%$ en el tratamiento con Hormonagro y del $24.4 \%$ en el tratamiento con Sábila al comparar el muestreo a los 45 y 60 días, mientras que en el testigo hubo un incremento del $63.6 \%$. Estos resultados dan indicios de que los tratamientos evaluados pueden tener un efecto negativo sobre el peso seco de raíces, principalmente en esta especie, sin embargo estadísticamente, los tratamientos en los dos muestreos fueron iguales, lo cual indica que existen otros factores que no fueron evaluados o no se conocen y que afectan la variable de respuesta, por lo cual es necesario profundizar en la investigación de esta especie.

Para la especie T. gigantea, los resultados fueron similares tanto en los tratamientos con Hormonagro ${ }^{\circledast}$ y el extracto de sábila como en el testigo sin enraizador. Para $S$. humboldtiana, el peso de raíces obtenido en el segundo muestreo, fue menor en todos los tratamientos, comparados con el registrado en el primer muestreo. 
Tabla1. Peso seco de raíces $(\mathrm{g})$ de tres especies vegetales tratadas con dos enraizadores, evaluadas 45 y 60 días después de la siembra.

\begin{tabular}{|c|c|c|c|c|c|c|c|}
\hline \multirow{5}{*}{$\begin{array}{l}\stackrel{\mathscr{\Xi}}{ت} \\
\stackrel{\tilde{Z}}{+}\end{array}$} & \multirow{2}{*}{ Enraizador } & \multicolumn{2}{|c|}{ Gliricidia sepium } & \multicolumn{2}{|c|}{ Trichanthera gigantea } & \multicolumn{2}{|c|}{ Salix humboldtiana } \\
\hline & & Media & C.V.\% & Media & C.V.\% & Media & C.V.\% \\
\hline & Hormonagro & $1,01 \mathrm{a}$ & 29,11 & $0,68 \mathrm{a}$ & 11,28 & $0,31 \mathrm{a}$ & 22,24 \\
\hline & Sábila & $0,90 \mathrm{a}$ & 44,91 & $0,59 \mathrm{ab}$ & 19,08 & $0,29 \mathrm{a}$ & 18,99 \\
\hline & Control & $0,55 \mathrm{a}$ & 35,67 & $0,48 \mathrm{~b}$ & 35,67 & $0,30 \mathrm{a}$ & 17,21 \\
\hline \multirow{5}{*}{ 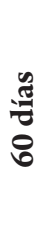 } & Fnmoinador & \multicolumn{2}{|c|}{ Gliricidia sepium } & \multicolumn{2}{|c|}{ Trichanthera gigantea } & \multicolumn{2}{|c|}{ Salix humboldtiana } \\
\hline & 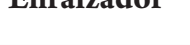 & Media & C.V.\% & Media & C.V.\% & Media & C.V.\% \\
\hline & Hormonagro & $0,73 \mathrm{a}$ & 37,95 & $0,69 \mathrm{a}$ & 38,10 & $0,25 \mathrm{a}$ & 59,83 \\
\hline & Sábila & $0,68 \mathrm{a}$ & 53,76 & $0,53 \mathrm{a}$ & 39,95 & $0,25 \mathrm{a}$ & 34,23 \\
\hline & Control & $0,90 \mathrm{a}$ & 45,35 & $0,44 \mathrm{a}$ & 33,27 & $0,20 \mathrm{a}$ & 17,36 \\
\hline
\end{tabular}

La mortalidad en todas las especies fue ocasionada principalmente por efectos de un exceso de humedad en el sustrato en que se realizó el experimento. Durante el primer muestreo, las mayores pérdidas (11.1\%) se presentaron en la especie G. sepium tratada con Hormonagro y las menores en las especies S. humboldtiana, tratada con Hormonagro y $T$. gigantea tratada con Sábila, pero, en este mismo tratamiento ( $T$. gigantea con sábila), se registraron las mayores pérdidas (11.1\%) en el segundo muestreo, mientras que las menores se registraron en la especie $S$. humboldtiana sin enraizador y en la especie G. sepium con Hormonagro. Sin embargo, el análisis estadístico muestra que no hay un efecto de los tratamientos ni de la especie sobre la mortalidad de estacas. (Tabla 2).

\section{Discusión y conclusiones}

En éste ensayo, las especies G. sepium y $S$. humboldtiana no respondieron de forma diferencial a la aplicación de los enraizadores, mientras que en $T$. gigantea pudo observarse un efecto diferencial 45 y 60 días después de la siembra con la aplicación de Hormonagro ${ }^{\oplus}$ y extracto de sábila, evidenciado por un mayor peso seco de raíz en comparación con el testigo.

La respuesta de las tres especies tratadas con enraizador de síntesis y con enraizador natural es similar, sin embargo el extracto de sábila mostró un buen efecto sobre el enraizamiento de las tres especies, siendo más notorio sobre S. humboldtiana, 60 días

Tabla 2. Peso seco de raíces $(\mathrm{g})$ de tres especies vegetales tratadas con dos enraizadores, evaluadas 45 y 60 días después de la siembra.

\begin{tabular}{ccccccc}
\hline & \multicolumn{3}{c}{ 45 días } & \multicolumn{3}{c}{ G0 días } \\
\hline Enraizador & G. sepium & T.gigantea & S.humboldtiana & G. sepium & T. gigantea & S. humboldtiana \\
\hline Hormonagro & 1.11 & 4.4 & 2.2 & 2.2 & 6.7 & 4.4 \\
Sábila & 8.9 & 2.2 & 4.4 & 8.9 & 11.1 & 4.4 \\
Control & 6.7 & 4.4 & 6.7 & 8.9 & 6.7 & 0 \\
\hline
\end{tabular}


después de la aplicación, lo que confirmaría su potencial para usarse como sustituto de enraizadores de síntesis en la propagación de ésta especie

La especie que registró en promedio el peso de raíces más alto fue G. sepium, seguido por la especie $T$. gigantea y por $S$. humboldtiana, independientemente de los tratamientos. Esta condición sumada a la resistencia de las raíces, podría indicar que G. sepium es una especie promisoria para ser utilizada en obras de bioingeniería para el control de erosión, aunque debe tenerse en cuenta que $G$. sepium parece responder de manera negativa a la aplicación de sustancias promotoras de enraizamiento, las cuales provocan un efecto de reducción del peso seco de la raíz a medida que pasa el tiempo, sin embargo las relaciones ligadas a éste efecto aun se desconocen.

El grosor y abundancia de raíces variaron entre tratamientos siendo las raíces de las estacas tratadas con el enraizador químico más abundantes y delgadas, mientras que las de las estacas tratadas con cristal de sábila fueron más gruesas y menos densas. El exceso de humedad del sustrato afecta negativamente la tasa de crecimiento y desarrollo de raíces, evidenciado porla reducción en peso seco entre un muestreo, a la vez que propicia el ataque de hongos y bacterias, lo cual influye finalmente sobre el desarrollo radicular de la planta. Salix humboldtiana presentó mayor tolerancia a las condiciones de encharcamiento por lo que puede considerarse una especie importante en la recuperación de suelos con mal drenaje. La mortalidad no estuvo relacionada con los tratamientos ni con las especies sino con las condiciones ambientales y exceso de humedad en el sustrato de siembra. Por esta razón, es necesario profundizar en la investigación de éstas especies en diferentes condiciones ambientales a fin de determinar los factores limitantes en el enraizamiento de estacas.

\section{Literatura citada}

Ángel, E. 1990. El manto de la tierra, flora de los andes, Guía de 150 especies de la flora andina, Ediciones Lerner Ltda., Bogotá, Colombia, 334pp

Caballero, JM \& C del Rio. 1999. Métodos de multiplicación C, Mundi-Prensa, Madrid. $112 \mathrm{pp}$

Colorado, SA. 2001. Establecimiento de una cobertura arbórea para la recuperación de un suelo erosionado utilizando seis especies forestales. Corporación Autónoma Regional del Valle del Cauca (CVC), Cali. Colombia. 8pp.

CONIF. 1986. Resultados del comportamiento de especies forestales plantadas en líneas de enriquecimiento en bajo Calima, San José del Guaviare y Tumaco. Corporacion nacional de investigacion y fomento forestal-CONIF. Bogotá, Colombia. 33pp.

Cornejo, P. 1997. Propagación vegetativa en olivo (Tesis Ingeniero Agrónomo). Universidad de Concepción Facultad de Agronomía, Concepción. Chile, 25pp.

Duran, F. 2007. Agricultura de las Américas, Instituto Colombiano agropecuario (ICA), Grupo Latinos Editores Ltda., Santa Fe de Bogotá, Cundinamarca. 968pp.

van Eijk-Bos, C. 1986. Barreras vivas de / Gliricidia sepium/ (Jacq.), Steud (Matarratón) y su efecto sobre la pérdida de suelo en terrenos de colinas bajas de Urabá (Colombia), Bogotá (Colombia), CONIF. 16pp.

Greenway, R. 1987. Vegetation and slope stability, Anderson, M., G. Jhon Wiley and Sons ed., New York. 230pp.

Hartmann, HT \& DE Kester. 1998. Propagación de plantas, principios y práctica, VI edición, Editorial Continental, Mexico. 760pp.

Hoffman, AG \& L Veghazi. 1992. Plantas Medicinales de uso común en Chile, Fundación Clauidio Gay, Santiago de Chile. 273pp.

Kramm, C. 1987. Propagación Vegetativa de cuatro especies arbustivas nativas con posibilidades ornamentales, (Tesis lisenciado en agronomía). Universidad Autral de Chile. 50pp.

Lal, R. 1994. Soil quality and agricultural sustainability. En: LAL, R. (Ed.) Soil quality and agricultural sustainability. p 3-12. Ann Arbor Press, Michigan, USA.

Llano, E. 1952. Propagación de plantas. Bogotá Colombia, Colinagro, 157pp.

Ospina, S \& RE Murgueitio. 2002. Tres especies vegetales promisorias: nacedero, botón de oro y bore. Convenio Andrés Bello-CAB, Cali, Valle del Cauca, Colombia. 302pp.

Parent, G. 1989. Guía de reforestación. Corporación para la Defensa de la Meseta de Bucaramanga, CDMB, Agencia Canadiense para el Desarrollo Internacional ACDI, Bucaramanga, Colombia. 214pp.

Rivera,JH.2001. Manejoy estabilización de taludes en zonas de laderas mediante tratamientos bioingenieriles. Avances Técnicos CENICAFE, Chinchiná, Colombia. 9pp,

Rivera, JH \& JA Sinisterra. 2006. Uso social de la bioingeniería para el control de la erosión 
severa. CIPAV, CVC, Cali, Colombia, 110pp.

Rivera, JH. 2002. Tratamientos biológicos y de bioingeniería para la prevención y control de erosión y movimientots masales en zonas de ladera colombiana, Cenicafé, Chinchiná, Colombia. $11 \mathrm{pp}$.

Rodríguez, J \& VM Nieto. 2002. Aplicación de los métodos de estacas e injertos para la propagación vegetativa de Cordia alliodora (Ruíz Pavón) Oken y Tabebuia rosea (Bertol.), DC. CONIF. Bogotá, Colombia. 61pp

Ross, C \& F Salisbury. 1994. Fisiología Vegetal, Iberoamericana, México. 759pp.

Rubio, LG \& PT Solorzano. 1996. Validación de un sistema agroforestal comunitario en cercas vivas de matarratón (Gliricidia Sepium), en la vereda Mapurá municipio de Quinchía, Risaralda. CARDER, Universidad del Tolima, Facultad de Ingeniería Forestal y Facultad de Medicina Veterinaria y Zootecnia, Ibagué. 165pp

Ruiz, L. 1998. Efecto de la aplicación de AIB y época de recolección sobre el enraizamiento de estacas semileñosas. Trabajo de Grado. Universidad Católica de Valparaíso. Facultad de Agronomía, Quillota. 46pp.

Schiechtl, HM. 1980. Bioengineering for land reclamation and conservation. University Of Alberta Press. Edmond, Alberta, Canada. 404pp.
Suarez, J. 1998. Deslizamientos y estabilidad de taludes en zonas tropicales, Universidad Industrial de Santander, Bucaramanga, (Colombia). 546pp.

Suárez, J. 2002. La Bioingeniería en el control de Erosión, Universidad Industrial de Santander, Bucaramanga. 23pp

Trujillo, NE. 1989. Producción sexual y vegetativa. Terie Técnica No. 1. Inforagro, Santa fe de Bogotá (Colombia). 157pp.

Urrego, B \& A Marín. 1997. Avances en la propagación del nogal cafetero Cordia alliodora a través de estacas enraizadas. SMURFIT Cartón de Colombia. Cali, Colombia 10pp.

Vázquez-Yanes, C, AI Batis-Muñoz, MI AlcocerSilva, M Gual-Díaz \& C Sánchez-Dirzo. 1999. Árboles y arbustos potencialmente valiosos para la restauración ecológica y la reforestación. Reporte técnico del proyecto J084. CONABIOInstituto de Ecología, UNAM.

Villegas, LC. 1984. Evaluación de dos promotores hormonales en tres dosis diferentes cada una en la reproducción asexual de Theobroma Cacao L. en dos ambientes diferentes, (Tesis Ingeniero Agrónomo). Universidad de San Carlos, Guatemala. 89pp.

Westwood, M. 1982. Fruticultura de zonas templadas, Mundi-Prensa, Madrid. 461pp. 